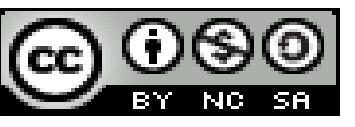

\title{
Una mirada acerca de restauraciones cerámicas
}

\author{
A look at ceramic restorations
}

\section{Una mirada sobre de restauraciones cerámicas}

\author{
Alex X. Bravo-Rodríguez ${ }^{\mathrm{I}}$ \\ alex.bravor@ug.edu.ec \\ Marisela S. Villarreal-Salazar II \\ marisela.vs@ug.edu.ec \\ María F. Paredes-Baidal III \\ maria.paredesb@ug.edu.ec
}

Recibido: 29 de octubre de 2018 *Corregido: 22 de noviembre de 2018 * Aceptado: 27 de diciembre de 2018

I. Magíster en Gerencia de Salud para el Desarrollo Local, Especializacao em Dentistica Restauradora, Odontólogo, Docente de la Universidad de Guayaquil, Guayaquil, Ecuador.

II. Especialista en Rehabilitación Oral, Odontólogo, Docente de la Universidad de Guayaquil, Guayaquil, Ecuador

III. Especialista en Dentistica Restauradora, Odontólogo, Docente de la Universidad de Guayaquil, Guayaquil, Ecuador 


\title{
Resumen
}

En la actualidad hay una creciente demanda de los pacientes por la estética, término muy subjetivo y que sufre grandes cambios según la época y el medio socio cultural al que nos refiramos. Las restauraciones elaboradas con porcelana ocupan un lugar central en la Odontología Restauradora y Rehabilitadora Estética. Se realizó una revisión bibliográfica del tema propuesto abordándose aspecto como: adhesión de los fragmentos cerámicos, sus propiedades, clasificación, indicación y por último se presentan unas pautas para orientar al profesional en la toma de decisiones.

Palabras claves: estética dental; cerámica composición química y técnica de confección

\begin{abstract}
A look at restorations At present there is a growing demand of patients for aesthetics, a very subjective term and that undergoes great changes according to the time and the socio-cultural environment to which we refer. The restorations made with porcelain occupy a central place in the Restorative Dentistry and Aesthetic Rehabilitation. A bibliographic review of the proposed theme was carried out, addressing aspects such as: adhesion of the ceramic fragments, their properties, classification, indication and finally some guidelines are presented to guide the professional in the decision making process.
\end{abstract}

Keywords: dental aesthetics; ceramics chemical composition and manufacturing technique

\section{Resumo}

Una mirada about de restauraciones Na verdade, a razão de uma subcoleção de pacientes pela estética, um período muito alto e as grandes razões para a época e o meio sócio-cultural al que nos refiramos. Las restauraciones elaboradas con porcelana ocupam uma localização central na Odontología Restauradora y Rehabilitadora Estética. A realizar uma revisão bibliográfica do tema proposital abordar o aspecto como: adesão dos fragmentos cerâmicos, propiedades, clasificación, indicación y por último se presentam unas pautas para orientar a profesional en la toma de decisiones.

Palavras-chave: estética dental; cerámica composición química y técnica de confección 


\section{Introducción}

El hombre se ha preocupado por mantener su apariencia física agradable a los demás durante la existencia de la humanidad, uno de los aspectos más interesantes de la fisonomía humana es la sonrisa, por esto, se considera de vital importancia el buen cuidado y mantenimiento del sector anterosuperior de la dentadura por ser la parte más expuesta. (Cedeño Intriago E . 2015)

Es mayoritariamente ver en hombres y mujeres piezas dentales del sector anterosuperior en mal estado de conservación o con deformaciones, lo cual torna a la sonrisa de esas personas una imagen desagradable o por lo menos no favorable; esta situación para quienes la sufren constituye un problema que limita su participación en la sociedad, muchos de ellos se cohíben sintiéndose por esto poco atractivos y se inhiben de emprender acciones en las que de otro modo podrían ser exitosos. (Cedeño Intriago E. 2015)

El sector anterosuperior de la dentadura humana está comprendido por las piezas dentales que se encuentran entre los dos caninos o premolares, parecería que estos dientes son más sensibles o menos resistentes que los demás, porque son los que muestran deterioro más temprano o malformaciones y defectos congénitos como: dientes mellados y fracturados, dientes conoides, dientes muy finos, dientes muy cortos o llenos de manchas muy intensas. . (Cedeño Intriago E. 2015)

La reconstrucción de los dientes afectados por enfermedades, traumatismos, defectos congénitos $u$ otras problemáticas es la función de la Operatoria Dental, procurando mantener y/o restaurar la forma, la función y estética, así como la integridad fisiológica del diente en relación armoniosa con la estructura dental remanente, y los tejidos blandos del sistema estomatognático. (Osores Ibáñez. J .2013)

Con referencia a lo anterior, la tarea de devolver la funcionalidad a un diente afectado se realiza utilizando materiales artificiales, los cuales pueden ser utilizados como restauraciones directas o indirectas. La directa es aquella en la que se trabaja el material preparando una masa plástica que se lleva a la boca del paciente y se coloca en la zona a restaurar; la indirecta es aquella en la que se da forma definitiva al material restaurador fuera de la boca del paciente. (Osores Ibáñez. J .2013) 
A estos problemas se han dado soluciones tradicionales no recomendables, desde extracción de piezas hasta cirugías costosas, dolorosas y temporales. Hoy se presenta la alternativa de una verdadera solución con la aplicación de carillas de porcelana o láminas de cerámica que por su espesor son parecidas a una lente de contacto para ojos, que corrigen el mal sin causar daños externos con excelente resultado estético, se colocan sobre los dientes y forman una pieza firme y resistente.

\section{Desarrollo}

Según Murillo Cordero, Luis F (2010), adherir fragmentos de dientes a tejido remanente fracturado por medio de la técnica de acondicionamiento ácido a esmalte y dentina, con sistemas adhesivos, es una práctica clínica odontológica ya aprobada.

En cuanto a la rehabilitación oral, Corts J, Abella R. (2013) plantea que debe tenerse siempre como requisito previo, una planificación diagnóstica funcional y estética ordenada y una programación secuenciada de procedimientos, cuya complejidad variará según las exigencias del caso. Esa programación terapéutica secuencial es de gran ayuda para optimizar resultados y que el tratamiento sea eficaz y eficiente.

Donde se utilice el procedimiento de la Adhesión o unión de tejido dental a tejido dental o de un material basado en resina a tejido dental, participan una serie de factores sobre los sustratos: esmalte y dentina. Cada uno de ellos presenta características biológicas diferentes y su comportamiento durante la Adhesión es diferente. El efecto de unión sobre estos sustratos es relevante pero se debe poner extrema atención en el acondicionamiento del sustrato esmalte, pues a veces se deja de lado como un procedimiento extremadamente mecánico y no aplicándole su debida atención, siendo la llave del éxito en la Adhesión de restauraciones adhesivas estéticas. Como bien lo establece Lopes (2007) "la unión al esmalte es un procedimiento simple en la práctica dental, sin embargo algunos detalles pueden influenciar su durabilidad." La técnica de grabado ácido al esmalte es un tratamiento químico que amplía la topografía del esmalte, cambiando la baja energía de superficie a una superficie más susceptible a la Adhesión y su relación o contacto con resinas fluidas de baja viscosidad interactúan en las microporosidades por atracción capilar formando prolongaciones de resina dentro de esas microporosidades, (Lopes, 2007) estableciendo así un mecanismo de selle marginal en las 
restauraciones y retención de los materiales basados en resina por enlaces químicos. El profesional debe conocer el comportamiento y la relación del esmalte acondicionado con los sistemas adhesivos del tipo primeros hidrofílicos como los factores que influencian la durabilidad de la fuerza de unión: viscosidad de los sistemas adhesivos, concentración del ácido, tiempo del grabado ácido, tipo de esmalte, limpieza del esmalte y contaminación del esmalte (Lopes, 2007, Nakabayashi, 1998).

La fijación del fragmento al diente remanente llega a ser posible solamente con el mejoramiento de los sistemas adhesivos, técnicas adhesivas y materiales restaurativos. Los conceptos actuales de adhesión y el conocimiento amplio de la biología de la dentina logran efectos de incalculable valor en cuanto a la fuerza de adhesión y sustancial mejoría en la disminución de micro infiltración, aunque la expectativa por la longevidad de la adhesión se mantiene. Muy relacionado a estos medios adhesivos, se encuentra la adecuada adaptabilidad del fragmento al diente remanente. Murillo Cordero, Luis $\mathrm{F}$ (2010),

\section{Restauraciones cerámicas}

Las cerámicas son definidas como materiales inorgánicos no metálicos, fabricados por el hombre por calentamiento de cristales a elevadas temperaturas. Desde un punto de vista físico, las cerámicas y vidrios son clasificados como materiales de alta resistencia a la compresión, pero baja resistencia a la tracción por lo que pueden ser fracturados a tensiones muy bajas. Así, las cerámicas dentales muestran resultados catastróficos a la flexión en comparación con otros materiales dentales, como los metales, por ejemplo, los cuales presentan elasticidad y ductilidad gracias a la naturaleza interatómica de la nube de electrones compartidos, los que pueden fácilmente trasladar la energía aplicada. Lo que les confiere, además la capacidad de ser excelentes conductores térmicos y eléctricos, lo que no ocurre en las cerámicas. Por otro lado, los sistemas cerámicos, al ser utilizados en el reemplazo morfológico y funcional de los tejidos dentarios dañados, son considerados altamente estéticos, estables cromáticamente, resistentes a la abrasión y no generan reacciones alérgicas al ser comparadas con los metales. ( Saavedra, R., Iriarte, R, Moncada, G. 2014.), (Rizkalla AS, Jones DW 2004) 
La perfecta imitación de los tejidos duros dentales en relación a la luz incidente sólo es posible con materiales que se comporten con ella de forma similar a la que presentan los dientes naturales en cuanto a translucidez, vitalidad, coloración, textura, grosor, etc.

Actualmente, hablar de restauraciones estéticas implica hablar de cerámica sin metal. Han sido tan importantes y revolucionarios los cambios y aportaciones en este campo en los últimos años que en la actualidad existen multitud de sistemas cerámicos. Todos ellos buscan el equilibrio entre los factores estéticos, biológicos, mecánicos y funcionales. Sin embargo, existen diferencias considerables entre ellos. Por lo tanto, para seleccionar la cerámica más adecuada en cada caso, es necesario conocer las principales características de estos materiales y de sus técnicas de confección. (Martínez Rus, Francisco, Pradíes Ramiro, Guillermo, Suárez García, Ma Jesús, \& Rivera Gómez, Begoña. (2007).

\section{Clasificación de los sistemas totalmente cerámicos}

Martínez Rus et al (2007) informa que a pesar de que las clasificaciones son totalmente artificiales, siempre nos ayudan porque permiten organizar mejor los conocimientos sobre una determinada materia. Por ello, vamos a agrupar los sistemas totalmente cerámicos en función de dos criterios: composición química y técnica de confección.

\section{Clasificación por la composición química}

Cerámicas feldespáticas: Constan de un magma de feldespato en el que están dispersas partículas de cuarzo y, en mucha menor medida, caolín. El feldespato, al descomponerse en vidrio, es el responsable de la translucidez de la porcelana. El cuarzo constituye la fase cristalina. El caolín confiere plasticidad y facilita el manejo de la cerámica cuando todavía no está cocida. Además, para disminuir la temperatura de sinterización de la mezcla siempre se incorporan «fundentes». Conjuntamente, se añaden pigmentos para obtener distintas tonalidades. Al tratarse básicamente de vidrios poseen unas excelentes propiedades ópticas que nos permiten conseguir unos buenos resultados estéticos; pero al mismo tiempo son frágiles y, por lo tanto, no se pueden usar en prótesis fija si no se «apoyan» sobre una estructura. Por este motivo, estas porcelanas se utilizan principalmente para el recubrimiento de estructuras metálicas o cerámicas. La existencia de una demanda de mayor estética en las

355 Vol. 5, núm. 1, enero, 2019, pp. 350-362

Alex X. Bravo Rodríguez, Marisela S. Villarreal Salazar, María F. Paredes Baidal 
restauraciones, se fue modificando la composición de las cerámicas hasta encontrar nuevos materiales que tuvieran una tenacidad adecuada para confeccionar restauraciones totalmente cerámicas. En este contexto surgieron las porcelanas feldespáticas de alta resistencia. Éstas tienen una composición muy similar a la anteriormente descrita. Poseen un alto contenido de feldespatos pero se caracterizan porque incorporan a la masa cerámica determinados elementos que aumentan su resistencia mecánica (100-300 MPa). (Martínez Rus et al (2007)

Cerámicas aluminosas: En la actualidad las cerámicas de alto contenido en óxido de aluminio se reservan únicamente para la confección de estructuras internas, siendo necesario recubrirlas con porcelanas de menor cantidad de alúmina para lograr un buen mimetismo con el diente natural. (Martínez Rus et al (2007)

Cerámicas circoniosas: Este grupo es el más novedoso. Estas cerámicas de última generación están compuestas por óxido de circonio altamente sinterizado (95\%), estabilizado parcialmente con óxido de itrio (5\%). El óxido de circonio $\left(\mathrm{ZrO}_{2}\right)$ también se conoce químicamente con el nombre de circonia o circona. La principal característica de este material es su elevada tenacidad debido a que su microestructura es totalmente cristalina y además posee un mecanismo de refuerzo denominado «transformación resistente». Este fenómeno descubierto por Garvie \& cols. En 1975 consiste en que la circonia parcialmente estabilizada ante una zona de alto estrés mecánico como es la punta de una grieta sufre una transformación de fase cristalina, pasa de forma tetragonal a monoclínica, adquiriendo un volumen mayor. De este modo, se aumenta localmente la resistencia y se evita la propagación de la fractura.

Esta propiedad les confiere a estas cerámicas una resistencia a la flexión entre 1000 y $1500 \mathrm{MPa}$, superando con un amplio margen al resto de porcelanas. Por ello, a la circonia se le considera el «acero cerámico». Estas excelentes características físicas han convertido a estos sistemas en los candidatos idóneos para elaborar prótesis cerámicas en zonas de alto compromiso mecánico. A este grupo pertenecen las cerámicas dentales de última generación: DC-Zircon ${ }^{\circledR}$ (DCS), Cercon ${ }^{\circledR}$ (Dentsply), InCeram $^{\circledR}$ YZ (Vita), Procera ${ }^{\circledR}$ Zirconia (Nobel Biocare), Lava ${ }^{\circledR}$ (3M Espe), IPS e.max ${ }^{\circledR}$ Zir-CAD (Ivoclar), etc. Al igual que las aluminosas de alta resistencia, estas cerámicas son muy opacas (no 
tienen fase vítrea) y por ello se emplean únicamente para fabricar el núcleo de la restauración, es decir, deben recubrirse con porcelanas convencionales para lograr una buena estética.

El nuevo reto de la investigación es aumentar la fiabilidad de las actuales cerámicas monofásicas aluminosas y circoniosas. Recientemente, se ha demostrado que la circonia tetragonal metaestable en pequeñas proporciones (10-15\%) refuerza la alúmina de forma significativa ${ }^{4}$. Estos «composites» altamente sinterizados alcanzan unos valores de tenacidad y de tensión umbral mayores que los conseguidos por la alúmina y la circonia de forma individual. Además, tienen una adecuada dureza y una gran estabilidad química. Así pues, estos biomateriales de alúmina-circonia se presentan como una alternativa a tener en cuenta en el futuro para la confección de restauraciones cerámicas. (Martínez Rus et al (2007)

Así mismo Martínez Rus et al (2007) en su artículo platnea la clasificación por la técnica de confección:

La forma de confección en el laboratorio de las cerámicas es bastante útil y representativa, y para determinar su clasificación se dividen en tres grupos: condensación sobre muñón refractario, sustitución a la cera perdida y tecnología asistida por ordenador.

Condensación sobre muñón refractario: Esta técnica se basa en la obtención de un segundo modelo de trabajo, duplicado del modelo primario de escayola, mediante un material refractario que no sufre variaciones dimensionales al someterlo a las temperaturas que requiere la cocción de la cerámica. La porcelana se aplica directamente sobre estos troqueles termo resistentes. Una vez sinterizada, se procede a la eliminación del muñón y a la colocación de la prótesis en el modelo primario para las correcciones finales. Son varios los sistemas que utilizan este procedimiento: Optec-HSP ${ }^{\circledR}$ (Jeneric), Fortress $^{\circledR}$ (Myron Int), In-Ceram ${ }^{\circledR}$ Spinell (Vita), etc. (Martínez Rus et al (2007)

Sustitución a la cera perdida: Este método está basado en el tradicional modelado de un patrón de cera que posteriormente se transforma mediante inyección en una estructura cerámica, tal y como clásicamente se efectúa con el metal. (Martínez Rus et al (2007)

Tecnología asistida por ordenador: Hoy en día, la tecnología CAD-CAM (Computer Aid Design Computer Aid Machining) nos permite confeccionar restauraciones cerámicas precisas de una forma 
rápida y cómoda. Todos estos sistemas controlados por ordenador constan de tres fases: digitalización, diseño y mecanizado. Gracias a la digitalización se registra tridimensionalmente la preparación dentaria. Esta exploración puede ser extraoral (a través de una sonda mecánica o un láser se escanea la superficie del troquel o del patrón) o intraoral (en la que una cámara capta directamente la imagen del tallado, sin necesidad de tomar impresiones). Estos datos se transfieren a un ordenador donde se realiza el diseño con un software especial. Concluido el diseño, el ordenador da las instrucciones a la unidad de fresado, que inicia de forma automática el mecanizado de la estructura cerámica. Los sistemas más representativos son $\operatorname{Cerec}^{\circledR}$ (Sirona), Procera ${ }^{\circledR}$ (Nobel Biocare), Lava ${ }^{\circledR}$ (3M Espe), DCS ${ }^{\circledR}$ (DCS), Cercon ${ }^{\circledR}$ (Dentsply), Everest ${ }^{\circledR}$ (Kavo), Hint-Els ${ }^{\circledR}$ (Hint-Els), etc. Actualmente, no existe suficiente evidencia científica para determinar cuál es el mejor procedimiento. Sin embargo, en lo que si están de acuerdo la mayoría de los autores es que en el futuro, la tecnología CAD/CAM se impondrá a la técnica de confección manual. (Martínez Rus et al (2007)

Con las técnicas descritas se puede realizar el volumen completo de la restauración y luego proceder a su caracterización mediante maquillaje superficial; o se puede confeccionar la estructura interna y luego terminarla mediante la aplicación de capas de porcelana feldespática convencional. El maquillaje superficial se utiliza más en incrustaciones y carillas. Mientras que la estratificación de capas es el método ideal para coronas y puentes, ya que nos permite obtener mejores resultados estéticos porque el color se consigue desde las capas profundas. (Martínez Rus et al (2007)

Otros autores aseveran otras calcificaciones como:

Según el criterio de la temperatura de procesado: Según este criterio las porcelanas se clasifican en porcelanas de alta, media y baja fusión. Recientemente se ha ampliado la clasificación con otras porcelanas que se trabajan a temperaturas muy inferiores e incluso en frío. (Sorensen JA, Kang S-K, Torres TJ, Knode H 1998), (Álvarez-Fernández MA, Peña-López JM, González-González IR 2003)

Según la composición y características estructurales: cerámicas convencionales y modernas vitro cerámicas. (Álvarez-Fernández MA, Peña-López JM, González-González IR 2003)

Literaturas estudiadas refieren que los materiales cerámicos dentales deben presentar una serie de propiedades las cuales relacionamos: 
$\checkmark$ Propiedades ópticas de vitalidad, translucidez, brillo, trasparencia, color (posibilidad de incorporar pigmentos), reflexión de la luz y textura, lo que implica grandes posibilidades estéticas al mimetizar los dientes naturales. (McLean JW 1991)

$\checkmark$ Biocompatibilidad local y general. Son los que presentan el mejor comportamiento con los tejidos vivos.

$\checkmark$ Durabilidad y estabilidad en el tiempo tanto en integridad coronal como en su aspecto por la gran estabilidad química en el medio bucal.

$\checkmark$ Compatibilidad con otros materiales y posibilidad de ser adheridas y grabadas mediante los sistemas cementantes adhesivos actuales

$\checkmark$ Baja conductividad térmica con cambios dimensiónales más próximos a los tejidos dentarios naturales que otros materiales restauradores utilizados. (Oden A, Arvidson K, Engquist B 1999)

$\checkmark$ Radiolucidez: cualidad ésta muy interesante pues permite detectar posibles cambios en la estructura dentaria tallada como caries marginales y actuar precozmente especialmente en las porcelanas de alúmina densamente sinterizadas y en las feldespáticas. (Oden A, Andersson M, Krystek-Ondracek I 1998)

$\checkmark$ Resistencia a la abrasión debido a su dureza. Esta propiedad constituye una seria desventaja y un importante problema clínico cuando se opone a dientes naturales, pues limita las indicaciones y depende directamente de la dureza del material cerámico y de la aspereza del mismo al ocluir sobre las superficies dentarias. Actualmente se considera que la porcelana vitrificada de grano fino es menos abrasiva para el antagonista. (Álvarez-Fernández MA, PeñaLópez JM, González-González IR 2003)

$\checkmark$ Resistencia mecánica. Alta resistencia a la compresión, baja a la tracción y variable a la torsión, lo que las convierte en rígidas pero frágiles. Quizá sea este el más grave inconveniente que presentan, tanto es así que los mayores esfuerzos investigadores se han dirigido a dotarlas de mayor resistencia. (Álvarez-Fernández MA, Peña-López JM, González-González IR 2003)

$\checkmark$ Procesado simple y coste razonable: la realización de coronas de porcelana no es precisamente fácil de realizar lo cual lleva aparejado un coste elevado. Sin embargo la generalización y automatización de la técnica hacen suponer que a la larga se producirá un abaratamiento de 
los costo de producción. (Álvarez-Fernández MA, Peña-López JM, González-González IR 2003)

Relata Diatto Saavedra P (2016) en su investigación que las restauraciones confeccionadas en las nuevas porcelanas parecen ser una alternativa válida a las restauraciones convencionales metalcerámica, si bien, no las sustituyen completamente y no están exentas de problemas destacando principalmente el alto índice de fracturas.

En la década de los Vega del Barrio (1997) hace algunas referencias en el cierre de su investigación que la utilización de carillas de porcelana como procedimiento restaurador en alteraciones del color de los dientes, ofrece unos resultados satisfactorios a medio plazo, y las complicaciones que pueden surgir son escasas y fácilmente solucionables.

Martínez Rus et al (2007) en su artículo científico revela algunas consideraciones a tener en cuenta para la elección de la cerámica en función del caso que se presente:

$\checkmark$ Para realizar incrustaciones cerámicas, elegiremos las porcelanas feldespáticas, ya que son las únicas que nos permiten realizar restauraciones conservadoras manteniendo el binomio estética-resistencia.

$\checkmark \quad$ A la hora de elegir el sistema cerámico para confeccionar coronas en el sector anterior habrá que evaluar el color del sustrato.

$\checkmark$ En las coronas posteriores, el criterio que prima en la elección del material es la resistencia a la fractura. Por eso, elegiremos entre las cerámicas aluminosas o circoniosas, ya que sus propiedades mecánicas cumplen sobradamente con los requerimientos de estas restauraciones.

El desarrollo cerámico dental es actualmente imparable; surgen nuevos materiales así como innovadores métodos de trabajar las mismas o parecidas porcelanas. Las nuevas técnicas consisten en utilizar las distintas porcelanas aprovechando sus diferentes propiedades para el florecimiento de la estética dental.

\section{Referencias Bibliográficas}


Álvarez-Fernández MA, Peña-López JM, González-González IR, Olay-García MS. (2003) Características generales y propiedades de las cerámicas sin metal. RCOE; 8(5):525-546.

Cedeño Intriago E. (2015). Uso de laminados cerámicos por estética en el sector anterosuperior. Recuperado de http://repositorio.ug.edu.ec/bitstream/redug/11399/1/CEDE\%C3\%91Oeric.pdf

Corts J, Abella R .2013. Protocolos de cementado de restauraciones cerámicas. Acta odontológica. X (2). ISSN 1510-8139

Diatto Saavedra P (2016). Supervivencia clínica de las nuevas Cerámicas Dentales. Recuperado de https://idus.us.es/xmlui/bitstream/handle/11441/61382/TFG\%20PEDRO\%20ANTONIO\%20D IATTO\%20SAAVEDRA.pdf? sequence=1

Lopes GC. et al. (2007): Enamel acid etching: a review. Compendium. .28 (1); 18 -25

McLean JW. (1991). The science and art of dental ceramics. Oper Dent 1991;16:149-56.

Martínez Rus, Francisco, Pradíes Ramiro, Guillermo, Suárez García, Ma Jesús, \& Rivera Gómez, Begoña. (2007). Cerámicas dentales: clasificación y criterios de selección. $R C O E, 12(4), 253-$ 263. Recuperado en 01 de diciembre de 2018, de http://scielo.isciii.es/scielo.php?script=sci_arttext\&pid=S1138$123 \mathrm{X} 2007000300003 \& \operatorname{lng}=\mathrm{es} \& \mathrm{t} \operatorname{lng}=\mathrm{es}$.

Murillo Cordero, Luis F.(2010). Fractura de incisivos centrales superiores: Adhesión de fragmentos. Reporte de caso clínico. Revista Científica Odontológica, 6( 1), pp. 20-27 Colegio de Cirujanos Dentistas de Costa Rica San José, Costa Rica

Nakabayashi N. et al. Hybridization of dental hard tissues. Quintessence Publishing Co.Ltd.1998, cap. III-IV

Oden A, Arvidson K, Engquist B (1999). . Procera allceram bridges. Int J Prosthodont ;12: 452-5

Oden A, Andersson M, Krystek-Ondracek I (1998). Five-year evaluation of Procera AllCera crowns. J Prosthet Dent 1998;80:450-6 
Osores Ibáñez. J. (2013). Estudio comparativo in vitro del grado de microfiltración marginal de restauraciones de resina compuesta realizadas con el sistema adhesivo XP BOND ${ }^{\circledR}$ utilizando la técnica de Grabado Ácido Total y de Grabado Ácido Selectivo del Esmalte.” Recuperado de http://repositorio.uchile.cl/bitstream/handle/2250/117490/Osores_J.pdf?sequence=1

Rizkalla AS, Jones DW.(2004). Mechanical properties of commercial high strength ceramic core materials. Dent Mater; 20(2):207-12.

Sorensen JA, Kang S-K, Torres TJ, Knode H. (19989. Inceram fixed partial dentures: tree yearclinical trial results. Calif Dent Assoc J ;26:207-14

Saavedra, R., Iriarte, R, Moncada, G. (2014). Clasificación y significado clínico de las diferentes formulaciones de las cerámicas para restauraciones dentales Acta odontológica; 52(2)

Vega del Barrio J M. (1997) Estudio del comportamiento clínico de 194 laminas de porcelana como procedimiento restaurador. Valoración a tres años. Tesis doctoral .Recuperado de https://biblioteca.ucm.es/tesis/19972000/D/3/D3005101.pdf 
